\title{
Migración y refugio en Panamá
}

Kevin Evandro Sánchez Saavedra

Responsable de comunicación e investigación en el Servicio Jesuita para Refugiados-Panamá e investigador del Servicio Jesuita para Migrantes de Centroamérica. Ciudad de Panamá: Correo electrónico: ksanchezs@gmail.com

\section{Recibido: mayo de 2008 / Aceptado: junio de 2008}

ESTE ARTíCUlO SEÑALA LA REALIDAD ACTUAL DE LA MIGRACIÓN y el refugio en Panamá y su relación con la situación social, política y económica de este país. Brinda pistas para reconocer el incremento del flujo migratorio hacia el país.

Las investigaciones del Servicio Jesuita para Refugiados-Panamá sugieren que la presencia de refugiados e inmigrantes en Panamá es concebida por esta sociedad como un problema. El gobierno y los medios de comunicación lo traducen como un asunto de seguridad que debe resolverse de una manera selectiva que, aunque no oficialmente, queda resumida en la consigna: "Empresarios, turistas y pensionados, iSí! Solicitantes de refugio, iNo!".

Palabras clave: inmigrantes / migración / Panamá / refugiados / colombianos

\section{Introducción}

Este artículo señala la actual realidad de la migración y el refugio en Panamá y su relación con la situación social, política y económica del país. Brinda pistas para reconocer el incremento del flujo migratorio hacia el país. Resaltan dos puntos importantes. Primero, el cambio radical de la actual Ley de Migración a inicios de 2008, que implica transformaciones en el trato a inmigrantes y solicitantes de refugio en Panamá. Este cambio fue realizado mediante decisiones exclusivas del Ejecutivo. El debate que debió existir con organizaciones de la sociedad civil para discutir dichas transformaciones no estuvo incluido de forma responsable en la agenda de gobierno. Segundo, que la presencia de refugiados e inmigrantes en Panamá es concebida por la sociedad como un problema. El gobierno y los medios lo traducen como un asunto de seguridad que debe resolverse de una manera selectiva que, aunque no oficialmente, queda resumida en la consigna: "Empresarios, turistas y pensionados, iSí! Solicitantes de refugio, iNo!".

\subsection{La expansión urbana}

Panamá se establece como república independiente a partir de 1903, luego de separarse de Colombia. ${ }^{1}$ Buena parte de su desarrollo económico, social, cultural y político, desde aquel entonces hasta la actualidad, ha estado relacionado e influido directa o indirectamente por el Canal de Panamá y la presencia militar norteamericana durante todo el siglo XX. El país está dividido política y administrativamente en nueve provincias y cinco comarcas indígenas. ${ }^{2}$ Tiene una extensión territorial de 75,517 $\mathrm{Km}^{2}$. Limita al norte con el Mar 
Caribe, al sur con el Océano Pacífico, al este con la República de Costa Rica y al oeste con la República de Colombia. El Istmo se encuentra en las latitudes bajas, en la región tropical.

Actualmente cuenta con una población de poco más de 3 millones de personas. Más de la mitad de esta población reside en el área metropolitana (Ciudad de Panamá, San Miguelito, Chorrera y Colón), influida por todo lo relacionado con el Canal de Panamá y su zona adyacente. Según estimaciones de la Dirección Nacional de Estadística y Censo, a mediados de 2006 la población urbana alcanzaría el 64\% del total de la población nacional (de ese porcentaje, el 49.4\% son hombres y el 50.6\% mujeres). El 90\% de la población de la Provincia de Panamá es urbana y representa el 71.3\% del total de la población urbana del país.

La experiencia del Servicio Jesuita para Refugiados-Panamá (SJR-Panamá) en los últimos cinco años ha demostrado que la mayor cantidad de refugiados e inmigrantes ingresan al país vía aérea por el Aeropuerto Internacional de Tocumen (Ciudad de Panamá). Por tanto, a esta gran y creciente masa de población urbana (migrantes internos) en el área metropolitana se le suma ésta otra (migrantes externos). En los últimos 30 años ha habido un aumento significativo en la cantidad de migrantes externos y refugiados en el país. Según datos del Censo, el total de inmigrantes en 1980 era 34,567; en 1990 era 54,551; y en el 2000 era 68,886 personas. ${ }^{3}$ Las nacionalidades más representativas son: colombianos, chinos, dominicanos y mexicanos.

En Panamá habitan también siete pueblos indígenas (ngóbes, buglé, bribri, naso, emberá, wounaan y kuna) y representan cerca de un 10\% del total de la población. ${ }^{4}$ El último censo evidenció que casi la mitad de la población de cada pueblo indígena reside en áreas urbanas, contrario a su patrón de asentamiento en áreas rurales y fronterizas. Buena parte de esta población indígena urbana se ubica en sitios periféricos a la Ciudad de Panamá donde han establecido barrios indígenas. ${ }^{5}$ Según estimaciones hechas por el Programa de las Naciones Unidas para el Desarrollo (PNUD), la pobreza alcanza a cerca del 98\% de la población indígena. En áreas rurales y comarcales indígenas los niveles de vida son los más bajos del país. ${ }^{6}$

\subsection{Riqueza y pobreza}

Casi el 40\% de la población total del país es pobre; es decir, cuatro de cada diez personas (PNUD, 2003:59). Cerca de medio millón se encuentra en pobreza extrema. Para 2003, en áreas rurales la pobreza alcanzaba al 62.7\%, mientras que en las áreas urbanas a cerca del 20\%. Para agravar más la situación, el 54.1\% de los niños menores de 6 años vive en situación de pobreza y el $29.4 \%$ de ellos en pobreza extrema. ${ }^{7}$

En contraste con este panorama cuantitativo de pobreza, Panamá se ubica en el ranking mundial de los 57 países con índice de desarrollo humano (IDH) alto, y entre los ocho países de Latinoamérica con el IDH más alto detrás de Argentina, Chile, México, Costa Rica o Cuba. Como ya lo señaló el mismo PNUD, este índice general enmascara el desigual reparto de la riqueza, la educación, la sanidad o las diferencias existentes entre áreas urbanas y rurales. Zonas del país tienen un desarrollo casi igual al de países del primer mundo y otras, un IDH igual al de países africanos. 
Sin duda Panamá ha tenido, en los últimos años, un crecimiento económico sostenido y alto con respecto a otros países de Latinoamérica. Este año el producto interno bruto (PIB) creció hasta un 11.2\%. Se cuenta con un buen ambiente de negocios, circulación del dólar norteamericano, ampliación del Canal, importante inversión extranjera y ampliación de la infraestructura portuaria. La ciudad de Panamá muestra su costanera llena de grandes y lujosos edificios, en semejanza directa a Miami Beach. En los últimos dos, años el boom inmobiliario ha promocionado 100 megaproyectos que incluyen 20 rascacielos (grandes especulaciones en los precios y las ventas se han dado también) y viviendas para pensionados de hasta un millón de dólares. ${ }^{8}$ Sin embargo, el país mantiene un déficit habitacional de 200 mil viviendas y carece de la infraestructura mínima necesaria que conlleva la construcción de las obras de magnitud considerable.

Este panorama de contraste entre riqueza y pobreza ${ }^{9}$ demuestra claramente una alta desigualdad económica y social en el país. En 2004, la ex embajadora de Estados Unidos en Panamá, exageradamente alarmada, advirtió sobre esta realidad por la posibilidad de abonar el camino al terrorismo y la violencia. Dijo que cerca de 80 personas en Panamá controlan más del 50\% del PIB, que estimó en unos 12 mil millones de dólares. ${ }^{10}$

Se une a lo anterior las desigualdades que existen en el acceso a la justicia y la percepción generalizada de la corrupción que existe en el sistema judicial panameño. La gran mora judicial persiste en el país, principalmente para aquellos que carecen de los medios económicos necesarios para agilizar los trámites administrativos o judiciales. Existe pues una corrupción aislada y una corrupción sistemática en el sistema judicial, siendo mucho más peligrosa y menos atacada esta última. ${ }^{11}$ A pesar de esto, Panamá es imaginado aún como paraíso fiscal, económico y de seguridad no sólo por empresarios de otros países, transnacionales o pensionados, sino también por la masa de empobrecidos, excluidos y perseguidos de los demás países de América Latina. Muchos han escuchado sobre los supuestos 5,650 millones de dólares para la construcción del tercer juego de esclusas, sobre los 40 mil empleos directos e indirectos para su construcción o sobre el aparente bajo costo de la vida. ${ }^{12}$

\section{Incremento del flujo migratorio}

El flujo migratorio es constante, las salidas y entradas por el Aeropuerto Internacional de Tocumen se han ido incrementando año tras año. Sin embargo, debe distinguirse entre aquel que es producto del turismo (donde la inmigración norteamericana ocupa el primer lugar) y aquel que tiene que ver con la migración económica o la búsqueda de refugio.

La experiencia de trabajo del SJR-Panamá revela una mayor cantidad en el flujo de ciudadanos colombianos hacia Panamá. Debido a la situación de conflicto que se vive en Colombia, una gran parte de los que ingresan a Panamá se encuentra en situación de refugio. Sin embargo, la opinión pública y los gobiernos panameños han visto este flujo como una simple y llana inmigración económica. Las opiniones sobre ellos se encuentran prejuiciadas y frecuentemente se puede percibir prácticas discriminatorias. 
Cuadro 1. Registro de inmigrantes en Panamá por distintos puntos de entrada y salida: 2005-2007

\begin{tabular}{|c|c|c|c|c|c|c|}
\hline \multirow{3}{*}{ País } & \multicolumn{6}{|c|}{ Años } \\
\hline & \multicolumn{2}{|c|}{2005} & \multicolumn{2}{|c|}{2006} & \multicolumn{2}{|c|}{2007} \\
\hline & Entrada & Salida & Entrada & Salida & Entrada & Salida \\
\hline Argentina & 18,880 & 18,170 & 24,933 & 23,690 & 37,992 & 32,811 \\
\hline Bolivia & 2,513 & 2,512 & 2,947 & 2,886 & 2,587 & 2,235 \\
\hline Colombia & 134,197 & 130,730 & 156,496 & 146,622 & 209,138 & $\mathbf{1 7 8 , 8 9 5}$ \\
\hline Costa Rica & 73,664 & 63,322 & 95,230 & 84,328 & 117,924 & 103,564 \\
\hline Ecuador & 19,683 & 18,862 & 21,775 & 20,432 & 22,544 & 19,367 \\
\hline El Salvador & 13,946 & 12,428 & 15,471 & 14,801 & 16,126 & 13,076 \\
\hline Estados Unidos & 182,045 & 178,072 & 205,848 & $\mathbf{1 9 4 , 3 2 5}$ & 229,311 & 201,323 \\
\hline Guatemala & 17,761 & 16,926 & 21,467 & 19,933 & 23,483 & 20,920 \\
\hline Haití & 5,514 & 5,356 & 6,761 & 6,366 & 8,421 & 7,147 \\
\hline México & 28,837 & 27,301 & 34,299 & 32,216 & 44,990 & 38,927 \\
\hline Nicaragua & 15,721 & 14,496 & 19,126 & 18,231 & 22,379 & 19,578 \\
\hline Perú & 10,262 & 8,898 & 13,231 & 12,401 & 15,903 & 13,759 \\
\hline Rep. Dominicana & 7,995 & 7,601 & 10,282 & 9,744 & 12,479 & 10,779 \\
\hline & 19,494 & 19,340 & 31,604 & 29,655 & 108,539 & 91,157 \\
\hline
\end{tabular}

Fuente: Dirección Nacional de Migración y Naturalización

Nota: Actualizada hasta el 31 de diciembre de 2007.

Cuadro 2. Visas aprobadas y denegadas por país de nacionalidad: 2005-2007

\begin{tabular}{|l|r|r|r|}
\hline \multirow{2}{*}{\multicolumn{1}{|c|}{ País }} & \multicolumn{3}{|c|}{ Visas de Migrantes } \\
\cline { 2 - 4 } & \multicolumn{3}{|c|}{$\mathbf{2 0 0 5}-2007$} \\
\cline { 2 - 4 } & Aprob. & Deneg. & \multicolumn{1}{c|}{ Ttal. } \\
\hline Argentina & $\mathbf{1 8 8}$ & $\mathbf{6 5}$ & $\mathbf{2 5 3}$ \\
\hline Bolivia & 36 & 4 & 40 \\
\hline Colombia & $\mathbf{2 , 8 0 9}$ & $\mathbf{1 , 8 6 1}$ & $\mathbf{4 , 6 7 0}$ \\
\hline Costa Rica & $\mathbf{2 5 4}$ & $\mathbf{1 1 3}$ & $\mathbf{3 6 7}$ \\
\hline Ecuador & 149 & 67 & 216 \\
\hline El Salvador & 88 & 32 & 120 \\
\hline Estados Unidos & $\mathbf{4 9 5}$ & $\mathbf{1 7 1}$ & $\mathbf{6 6 6}$ \\
\hline Guatemala & 62 & 26 & 88 \\
\hline Haití & 27 & 49 & 76 \\
\hline México & $\mathbf{1 9 8}$ & $\mathbf{5 8}$ & $\mathbf{2 5 6}$ \\
\hline Nicaragua & 167 & 81 & 248 \\
\hline Perú & $\mathbf{3 0 9}$ & $\mathbf{1 9 5}$ & $\mathbf{5 0 4}$ \\
\hline Rep. Dominicana & $\mathbf{5 9 8}$ & $\mathbf{6 1 5}$ & $\mathbf{1 , 2 1 3}$ \\
\hline Venezuela & $\mathbf{6 0 8}$ & $\mathbf{1 2 8}$ & $\mathbf{7 3 6}$ \\
\hline
\end{tabular}

Cuadro 3. Cantidad de deportaciones por país: 2005-2007

\begin{tabular}{|l|r|r|r|r|}
\hline \multirow{2}{*}{\multicolumn{1}{|c|}{ País }} & \multicolumn{3}{|c|}{ Años } & \multirow{2}{*}{ Total } \\
\cline { 2 - 4 } & $\mathbf{2 0 0 5}$ & $\mathbf{2 0 0 6}$ & $\mathbf{2 0 0 7}$ & \\
\hline Argentina & 1 & 2 & 8 & 11 \\
\hline Bolivia & 3 & 3 & 0 & 6 \\
\hline Colombia & $\mathbf{6 9 7}$ & $\mathbf{8 0 8}$ & $\mathbf{1 0 7 6}$ & $\mathbf{2 5 8 1}$ \\
\hline Costa Rica & 7 & 13 & 13 & 33 \\
\hline Ecuador & $\mathbf{1 0 3}$ & $\mathbf{1 3 4}$ & $\mathbf{8 9}$ & $\mathbf{3 2 6}$ \\
\hline El Salvador & 16 & 10 & 4 & 30 \\
\hline Estados Unidos & $\mathbf{1 9}$ & $\mathbf{1 6}$ & $\mathbf{2 3}$ & $\mathbf{5 8}$ \\
\hline Guatemala & 13 & 9 & 27 & 49 \\
\hline Haití & $\mathbf{1 0}$ & $\mathbf{4 8}$ & $\mathbf{2 0}$ & $\mathbf{7 8}$ \\
\hline México & 5 & 9 & 20 & 34 \\
\hline Nicaragua & $\mathbf{4 0}$ & $\mathbf{4 6}$ & $\mathbf{4 6}$ & $\mathbf{1 3 2}$ \\
\hline Perú & $\mathbf{7 3}$ & $\mathbf{9 1}$ & $\mathbf{5 2}$ & $\mathbf{2 1 6}$ \\
\hline Rep. Dominicana & $\mathbf{4 3}$ & $\mathbf{4 7}$ & $\mathbf{4 7}$ & $\mathbf{1 3 7}$ \\
\hline Venezuela & 6 & 11 & 9 & 26 \\
\hline
\end{tabular}

Fuente: Sección de Asesoría Legal de la Dirección Nacional de Migración y Naturalización.

Nota: Actualizada hasta el 31 de diciembre de 2007. 
“iAve María, pues! Pa’ qué van a quitar el tapón del Darién si ya todos estamos acá (Paisas en Panamá)". La Cáscara, un programa televisivo nacional de burla, humor y sátira, colocó esta frase el 19 de marzo de 2005 como parte de su sección "El pensamiento de hoy". El 30 de marzo del mismo año, en la parada de buses de Paraíso, en San Miguelito (Ciudad de Panamá), un policía de tránsito le dice a un amigo conductor de bus colectivo: "sólo los que tengan mil dólares podrán conducir en el futuro, en el famoso transmilenio". Su amigo le responde: "entonces los que manejarán sólo serán colombianos". A finales de 2006, uno de los programas televisivos de mayor audiencia en Panamá fue Bailando por un sueño. En su primer día de difusión el presentador no solamente dio la bienvenida a una teleaudiencia compuesta por más de 3 millones de panameños sino que también a 100,000 colombianos y 300,000 chinos que, según él, habitan en el país. "Hay más de 80 mil paisas en el país", "Seguridad los atrae", son titulares de El Panamá América, periódico de circulación nacional que, el 28 de febrero de 2007, señala las causas por las que miles de colombianos salen de su país y menciona que las cifras reales siguen siendo una incógnita. Hace énfasis en que muchos vienen en busca de protección y que casi en su totalidad los solicitantes de refugio son colombianos.

De una u otra forma, estas cuatro apreciaciones seguramente son las mismas de muchos ciudadanos y ciudadanas de este país. Sin duda son inspiradas por la propia realidad. En la vida cotidiana del área metropolitana es común coincidir con ciudadanos colombianos. En las últimas décadas ha habido un aumento considerable y progresivo de ciudadanos colombianos. No solamente figuran en los titulares de los periódicos de circulación nacional, sino también en las cifras de solicitudes de visa o en las deportaciones, aunque en las cifras de deportados también sobresalen otras nacionalidades como: ecuatorianos, haitianos, nicaragüenses, peruanos y dominicanos.

En éstas cifras no aparecen los vejámenes y violaciones a los derechos humanos hacia los inmigrantes presentes en el país. Estos hechos no son sacados a la luz pública con mucha frecuencia. De hecho, muchos permanecen en el anonimato debido a que gran parte de los inmigrantes presentes en el país mantienen un estatus irregular. Debido al temor de ser detenidos y deportados, frecuentemente callan los malos tratos, las burlas, la explotación laboral, las extorsiones, entre otras muchas situaciones que atentan contra su dignidad. Muchos inmigrantes terminan asumiendo como algo normal estas prácticas discriminatorias o desconocen sus derechos inherentes, trascendentales que adquieren como seres humanos.

A raíz del primer incendio que se suscitó en Curundú (Ciudad de Panamá), una de las medidas adoptadas por el gobierno para supuestamente frenar la inseguridad, fue disminuir el tiempo de las visas de turista. De tres meses fue rebajado a un mes. La realidad de la migración y el refugio en Panamá, tal como sucede en otros países de América Latina, es tratada como un asunto de seguridad nacional. Se cierran fronteras, se limitan las entradas y se violentan libertades fundamentales. Cabe destacar que, desde 2006, el SJR-Panamá ha denunciado esta situación ${ }^{13}$ así como la persecución más directa a inmigrantes a partir de la segunda mitad de 2007. ${ }^{14}$ Durante 2007 el SJR-Panamá denunció también el aumento de la xenofobia y la discriminación contra inmigrantes y refugiados colombianos. ${ }^{15}$ 


\section{Realidad del refugio}

Como señalamos, el flujo de ciudadanos colombianos hacia Panamá, especialmente hacia la Ciudad, es visto como un asunto de inmigración económica y seguridad nacional por parte del gobierno panameño y la opinión pública. Son las personas de esta nacionalidad las que aparecen en las cifras de la Oficina Nacional para la Atención a los Refugiados (ONPAR) como los que mayormente solicitan refugio.

La atención hacia la realidad del refugio tiene su antecedente en la década de 1970 y 1980 cuando el gobierno militar de aquel entonces brindó protección a un buen número de refugiados centroamericanos. Sin embargo, es a partir de 1996 que se produce un recrudecimiento en el enfrentamiento de paramilitares, guerrilla y ejército en zonas fronterizas con Colombia, en el Departamento del Chocó, y se produce una entrada masiva de Población en Situación de Refugio (PSDR) hacia comunidades fronterizas en la Provincia de Darién. Inicialmente la política del gobierno fue la deportación, pero por presiones de organismos nacionales e internacionales estas malas prácticas pudieron frenarse. ${ }^{16}$

Desde entonces hasta la actualidad, el gobierno panameño, utilizando lo que establece el Decreto Ejecutivo $\mathrm{N}^{\circ} 23$ del 10 de febrero de 1998, ha reconocido una categoría subsidiaria al estatus de refugio. A las personas que se encuentran en Darién desde finales de la década de 1990 se les reconoce como Protegidos Humanitarios Temporales (PHT) o protegido provisional por razones humanitarias. El estatus se ha aplicado específicamente a la población en frontera. Hasta 2006, ONPAR mantenía un registro total de 829 PHT, viviendo en comunidades de frontera. Se supone que es un estatus temporal, pero la gente que lo ostenta tiene 10 años de permanecer en las comunidades en Darién. Se otorga en situaciones de "afluencia masiva", pero no se especifica cuántos deben ser para utilizar dicho concepto.

Los PHT son exactamente: 320 en Jaqué, 120 en Puerto Obaldía y 389 viviendo en Darién Centro (Matugantí, Sobiaquirú, Boca de Cupe, Yape, Pinogana, El Real, La Palma, Yaviza). ${ }^{17}$ A esta cifra debe agregarse 70 indígenas emberá, recientemente reconocidos como PHT, que residen en las comunidades de Tortuga, Riosito y Alto Playona. El principal problema por el que atraviesan estas personas -que es el agravante o la raíz de otras dificultades laborales, económicas, educativas, de salud y psicológicas- es el confinamiento al que son sometidos en las comunidades que los han acogido en Darién (comunidades receptoras). La práctica gubernamental restringe la libertad de movimiento de esta población. Para poder hacerlo, deben solicitar el permiso correspondiente a las autoridades locales (ONPAR, Oficina de Migración o la Policía). Éstas, a su vez, deben tramitar los permisos correspondientes en la Ciudad de Panamá. Debido a ello, por los problemas de comunicación en la región de frontera, la concesión de un permiso pueden tardar mucho tiempo o ser negado, a discreción de funcionarios de menor rango administrativo. El hecho de que la concesión de un permiso esté supeditado a la decisión de un funcionario público, ha generado preferencias o discriminación hacia algunos PHT. A criterio del funcionario, se otorga permiso a unos y se les niega a otros. No existen, pues, criterios establecidos o claros con respecto a este tema.

Aunque en zona de frontera la mayoría de la PSDR fue reconocida como PHT, a unas cuantas personas se les ha reconocido el estatus de refugio..$^{18}$ En estos casos, el reconocimiento 
fue otorgado luego de demostrar persecuciones directas por parte de paramilitares o la guerrilla, o en todo caso, después de demostrar el asesinato de parientes cercanos (algunos de estos casos fueron llevados por la Comisión de Justicia y Paz).

Aunque desde Colombia existe una gran movilización de la PSDR hacia Panamá, el reconocimiento del estatus de refugio es mínimo si se compara con otros países de Latinoamérica. Hasta 2006, según cifras de ONPAR, Panamá había reconocido el estatus de refugio a 947 personas. Esta cifra incluye a aquellos reconocidos desde décadas anteriores.

Cuadro 4. Refugiados reconocidos en Panamá, 2006

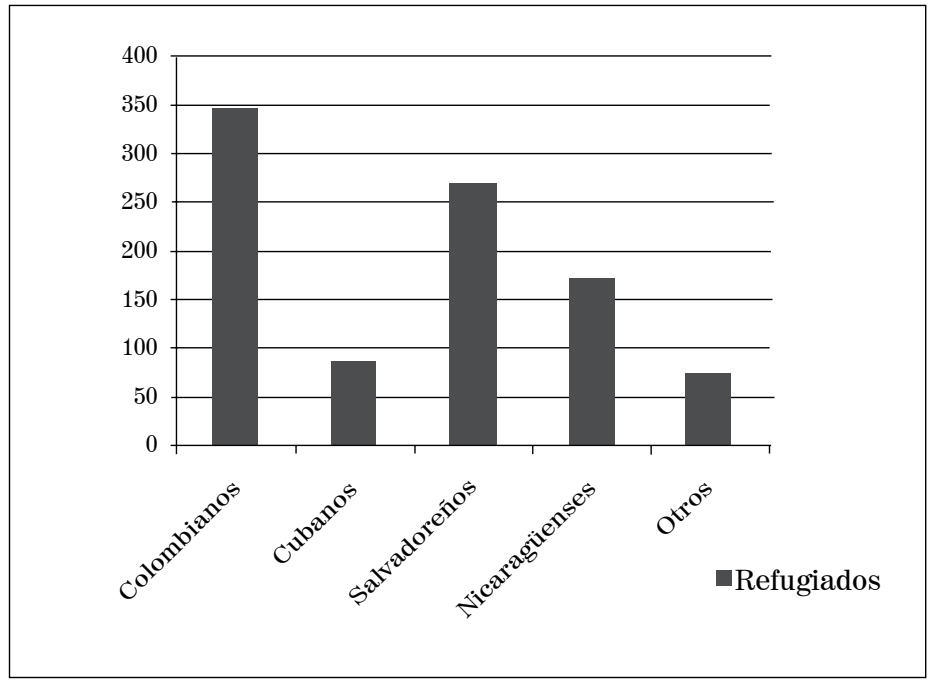

En el Cuadro 4 el registro de Otros incluye a: peruanos, haitianos, iraníes, libaneses y de los países de Europa del este. A este total de refugiados debe sumarse la cifra de 47 indígenas wounaan que ingresaron a Panamá en 2006 a través de Jaqué y actualmente residen en la comunidad de Vista Alegre (Darién). Un estudio realizado en 2003 (bajo el auspicio financiero del Alto Comisionado de las Naciones Unidas para los Refugiados ACNUR, la Embajada Sueca en Costa Rica y la Corporación Andina de Fomento) sugirió que la cantidad de personas colombianas en Panamá desplazadas por la violencia es de 12,104. Este total se distribuye de la siguiente manera: 4,712, en Colón; 2,026, en Darién; 5,223, en Ciudad de Panamá; y 143, en la Comarca Kuna Yala. Según el mismo estudio, esta población representaba el 6\% de cerca de 200,000 colombianos que han salido de Colombia a países fronterizos debido al conflicto (a Venezuela ha ido el 73\% y a Ecuador el 21\%) (FUMPADEM, 2004).

Nuestra experiencia, junto con las cifras que conocemos sobre la cantidad de ingresos registrados a través del Aeropuerto Internacional de Tocumen y por vía marítima, nos sugiere que la cantidad de personas en situación de refugio puede ser mucho más de las 12 mil que presenta este estudio. Además, el aumento de la cantidad de población colombiana 
en Panamá y países fronterizos de Colombia parece sugerir que el conflicto colombiano está lejos de solucionarse. ${ }^{19}$

De entrada, el problema que enfrenta la PSDR es precisamente la dificultad de su reconocimiento oficial por parte del estado panameño. El procedimiento administrativo para la solicitud de refugio es largo y el solicitante es sometido a un primer filtro donde se recibe o se deniega su solicitud. Otra dificultad con la que se enfrentan las organizaciones que defienden y acompaña a esta población es, por consecuencia, las estadísticas de las solicitudes denegadas y aceptadas. Como menciona el director del Centro de Investigación y Promoción de los Derechos Humanos (CIPDH): "nos preocupa la falta de estadísticas en cuanto a las personas que solicitan refugio y no son aceptadas en la etapa preliminar, como también aquellos casos que ni siquiera han realizado los trámites de la entrevista y pasan a engrosar las filas de los inmigrantes económicos al no tener acceso a la jurisdicción administrativa producto del desinterés del ONPAR. Lo que refleja el interés de no actualizar las cifras por parte del Gobierno Nacional y de mantener la compleja situación del refugio en un bajo perfil"20.

La PSDR que no es aceptada a trámite, como menciona este activista de derechos humanos, es catalogada como inmigrante económica. Debido a ello, puede ser sujeta de deportación. Es precisamente esta contradicción la que necesariamente debe visibilizarse ${ }^{21}$ y además solucionarse mediante la reforma del Decreto $\mathrm{N}^{0} 23$ o la propuesta de una Ley sobre Refugio.

Por su parte, aquellos admitidos a trámites también permanecen en indefensión y con serias vulnerabilidades económicas. Contradictoriamente, su admisión a trámite no les permite trabajar..$^{22}$ Dependen de la ayuda humanitaria que, en Ciudad de Panamá, únicamente brinda la Cruz Roja mediante fondos del ACNUR, o el SJR-Panamá. Pueden pasar muchos meses para que el caso sea considerado por la Comisión de Elegibilidad (entidad compuesta por autoridades ministeriales o de instituciones públicas vinculadas al tema, que reconoce el estatus de refugio). ${ }^{23}$ En ese lapso, las ayudas no son suficientes, la espera es larga y las necesidades mayores de acuerdo al tipo de solicitud (individual o familiar).

Además, el reconocimiento del estatus de refugio no brinda un pronto permiso de trabajo. El refugiado debe tramitar su permiso de trabajo en distintas instancias gubernamentales como: ONPAR, Dirección Nacional de Migración y Naturalización y el Ministerio de Trabajo. Este proceso puede tardar años. Existen refugiados que han tardado de uno a dos años para, finalmente, conseguir su permiso de trabajo que les garantice un mejor acceso al empleo. Otros problemas asociados al empleo tienen que ver con el acceso a la salud, a la educación y a la vivienda. Aunque resulta una dificultad tanto en frontera como en la ciudad, las condiciones estructurales de esta última, junto con una cultura más individualista y de dependencia total hacia el ingreso, hacen más vulnerables a los refugiados en la Ciudad.

En resumen, los problemas que enfrentan los refugiados en Panamá son:

- Aumento del rechazo hacia la población colombiana presente en Panamá. Existe un desconocimiento de la magnitud e impacto del conflicto armado en Colombia. Los inmigrantes y refugiados, principalmente los de nacionalidad colombiana, son 
estereotipados como delincuentes, narcotraficantes, prostitutas, guerrilleros, quita empleos, etc.

- Restricciones gubernamentales hacia el reconocimiento del estatus de refugio. El fenómeno de la inmigración y el refugio es entendido, principalmente, como un asunto de seguridad nacional. La PSDR puede ser sujeto de deportación, al ser rechazada su solicitud.

- En frontera, los PHT permanecen confinados a las comunidades receptoras. Esta situación plantea consecuencias en el empleo, la economía doméstica, la vida social, el acceso a la educación, la salud y estados emocionales. Los PHT carecen de los derechos que puede tener un refugiado reconocido oficialmente.

- Tanto los solicitantes de refugio como los refugiados reconocidos oficialmente tienen serios problemas para obtener permisos de trabajo. Los trámites son extensos y muy burocráticos. En la ciudad, esta situación genera mayores vulnerabilidades en el acceso al empleo, la educación, la salud y la vivienda.

\section{Perspectiva}

El flujo de personas hacia Panamá no parece detenerse y posiblemente continuará durante los próximos años. A este panorama de refugio e inmigración se une otro proceso regional: la situación política y social de Venezuela. Como quedó reflejado en los cuadros del acápite 2, la cantidad de venezolanos que ingresan a Panamá se va incrementando año tras año. De 2005 a 2007 las cifras aumentaron casi seis veces. Resulta importante comenzar a analizar la inserción y el nivel socioeconómico de esta población venezolana en Panamá. Se están dando los primeros pasos, no totalmente visibles, que van armando redes transnacionales de personas entre Panamá y Venezuela. La tensa situación de Venezuela, que parece haber disminuido luego del referéndum, podría traer consigo una posible masa de solicitantes de refugio.

¿Cuál es la respuesta del gobierno panameño frente a la realidad del flujo migratorio? Como se dijo, es visto como un asunto de seguridad. La política migratoria concebida es entonces de tipo selectiva. Se dieron los primeros pasos con la aprobación de la Ley $\mathrm{N}^{0} 1$, del 2 de enero de 2008, que concedió poderes extraordinarios al Ejecutivo para reformar la Ley de Migración.

La propuesta de Ley impulsada por el Ejecutivo, como en su momento se dijo para Mirada Global $^{24}$, determinaba quela definición general dela política migratoria panameña es potestad exclusiva del presidente Martín Torrijos y su gabinete de gobierno. Contrario a promesas y retórica gubernamental, quedaron excluidas de toda participación las organizaciones de la sociedad civil que desde hace varios años han estado brindando aportes sustanciales a las cuestiones de la migración y el refugio en Panamá.

El 22 de febrero de 2008 se aprobó el Decreto Ley $N^{\circ} 3$ que crea el Servicio Nacional de Migración, la Carrera Migratoria y dicta otras disposiciones. Uno de los aspectos más llamativos de esta Ley es la posibilidad de perseguir a los inmigrantes irregulares como si fueran delincuentes comunes. Para ello se contará con funcionarios capacitadores para tal fin, que posiblemente recibirán una formación policial. La Ley también discrimina por 
razones económicas. Podrán entrar al país y tendrán privilegios los turistas pensionados de países del norte, los rentistas retirados y los inversionistas. Mientras que aquellos inmigrantes expulsados de sus países por razones económicas, difícilmente podrán realizar los trámites correspondientes por lo oneroso que representan.

Muy probablemente los asuntos migratorios y de refugio, y su relación con el respeto de los derechos humanos, no sean de gran preocupación para la sociedad panameña, que se prepara para lo que será el inicio de un año electoral, donde todo se enfoca en candidaturas, campañas, torceduras de brazo, jugosos fondos electorales y generosos aportes empresariales. Sin duda, un carnaval electoral, escenario propicio para que posibles desmanes legales puedan pasar con facilidad.

\section{Notas}

1 Por 82 años el istmo panameño permanece unido a Colombia, luego de su independencia de España en 1821. Para detalles y referencia de estos sucesos puede verse: Beluche, 2006; Figueroa Navarro, 1978.

1062 Estas provincias son: Darién, Panamá, Colón, Coclé, Herrera, Los Santos, Veraguas, Chiriquí y Bocas del Toro. Las comarcas son: Kuna Yala, Ngóbe-Buglé, Emberá-Wounaan, Wargandí (kuna) y Madugandí (kuna).

3 Evidentemente estas cifras son inexactas porque seguramente sólo representan a aquellos migrantes que se encuentran con documentos o con residencia definitiva en el país. De la del 2000, 21,069 corresponde a la población colombiana. Entre la provincia de Panamá y Colón habita más del $80 \%$.

4 Todos estos pueblos tienen sus contrapartes en los países fronterizos. Ngóbes, nasos o teribes y bribris también habitan en zonas de Costa Rica, mientras que kunas, emberá y wounaan habitan regiones de Colombia.

5 Sólo en el corregimiento de Curundú (sitio donde el SJR-Panamá focaliza su labor), según el censo del 2000, la población indígena era de 1,717 personas (kunas 382 , ngóbes 26 , buglé 24 , teribe 4 , bokota (buglé) 7 , emberá 979 , wounaan 262 , bribri 27 , no declarado 6). En otras palabras, la población indígena en el corregimiento de Curundú representa el $9.1 \%$ de la población total.

6 Por ejemplo, en la Comarca Ngóbe-Buglé cerca de un 45\% de la población es analfabeta.

7 Ver estos y otros datos en www.undp.org.pa/portal/lang es/tabID 3507/DesktopDefault.aspx Consultado: 7/9/2007.

8 Con sólo colocar en el buscador electrónico Google las palabras "boom inmobiliario en Panamá" obtendrá cerca de 110,000 datos. Podemos rescatar de Camilo Andrés Sastoque, director de metrocuadrado.com, algunas de las razones para este boom inmobiliario: 1) los incentivos tributarios para la inversión extranjera, 2) la legislación especial para los pensionados, 3) el estatus que tiene el país como Iparaíso』 fiscal, 4) los instrumentos para establecer estructuras off shore, 5) el tránsito anual de 14 mil barcos, 6) la construcción del tercer juego de exclusas del Canal, y 7) la visita de cientos de miles de turistas, entre otros.

9 Un estudio de UNICEF llamó a esta realidad Los dos Panamá (Cordero, 2002).

10 Ver El Panamá América: 30/6/2004. Hughes y Quintero (2000) hablan de 76 personas y un PIB de 9 mil millones de dólares.

11 Para ver detalles de estos datos y conceptos de corrupción aislada y sistemática puede verse el reciente estudio llevado a cabo por la Fundación para el Debido Proceso (2007) (DPLF, por sus siglas en inglés).

12 Casi todos los servicios básicos (luz, agua, teléfono) han aumentado en más del $60 \%$ desde que la mayoría fue privatizada a partir de 1995. La canasta básica familiar actualmente puede llegar a más de 300 dólares mensuales. El precio del combustible también ha ido en aumento y junto con él muchos servicios y artículos de primera necesidad. Según el ranking mundial del costo de la vida, elaborado por Human Resource Consulting (MERCER), durante el 2005 la ciudad con el más bajo costo de vida en Latinoamérica era Asunción (Paraguay) y la más cara era San Juan (Puerto Rico). Curiosamente, Cuidad de Panamá se encontraba como ciudad más cara que Bogotá (Colombia), Quito (Ecuador), Caracas (Venezuela) y Sao Paulo (Brasil), entre otras.

13 Para ver esta nota puede revisar el siguiente enlace: www.jrs.net/alerts/index.php?lang=it\&sid=999 Consultado: 1/8/2006.

14 Para ver esta nota puede revisar los siguientes enlaces: www.jrs.net/alerts/index.php?lang=es\&sid=2576 Consultado: 1/9/2007. www.miradaglobal.com/index.php?option=com content\&task=view\&id=631\&ltemid=9\&nw=1\&lang=es\&section- 
=19 Consultado: 22/9/2007.

15 Para ver esta nota puede revisar el siguiente enlace: www.latinamerica.jrs.net/news/index.php?lang=es\&sid=1972 Consultado: 10/5/2007.

16 Para conocer otros detalles puede verse SERVIR N 41 , septiembre de 2007. Disponible en: www.jrs.net/pubs/servir/index. php?lang=es Consultado: 1/11/2007.

17 Para 2005, según cifras del Vicariato Apostólico de Darién, la población PHT estaba distribuida de la siguiente manera: 10 en Matugantí, 159 en Boca de Cupe, 61 en Yape, 8 en Pinogana, 2 en El Real, 8 en La Palma y 38 en Yaviza.

18 Para 2005, según cifras del Vicariato Apostólico de Darién, en Yape existían 10 refugiados reconocidos y en Jaqué 22.

19 Puede verse la reciente entrevista del director nacional del SJRM de Ecuador, Rodrigo Rovayo. Para él, el fenómeno del refugio se está invisibilizando, lo que conlleva una negación de los derechos humanos de la población en situación de refugio. Disponible en: www.miradaglobal.com/index.php?option=com content\&task=view\&id=702\&ltemid=9\&nw=1\&lang =es\&section-=19 Consultado: 28/11/2007.

20 Puede verse El Monitor Fronterizo, boletín informativo del CNR, № 1, noviembre de 2007. Disponible en: www.nrc.org. co/boletinfronterizo/html/panama cif.html Consultado: 20/11/2007.

21 Algunos esfuerzos menores se han hecho: “¿Inmigrantes sin documentos o refugiados?”. El Panamá América, Panamá, 4 de febrero de 2007. "¿Inmigrantes sin documentos o refugiados? II”. El Panamá América, Panamá, 5 de febrero de 2007.

22 Esta afirmación debe relativizarse, pues aunque legalmente no tienen permitido trabajar, la gran mayoría sí lo hace en pequeños negocios informales. De hecho, el SJR apoya iniciativas de este tipo mediante micro créditos.

23 Para conocer más detalles sobre la Comisión y la legislación panameña en materia de refugio puede verse: $\underline{w w w}$.asamblea. gob.pa/NORMAS/1990/1998/1998 157 1146.PDF Consultado: 7/3/2007, www.acnur.org/secciones/index.php?viewCat=88 Consultado: 9/9/2007.

24 Puede verse el siguiente enlace: $w w w . m i r a d a g l o b a l . c o m / i n d e x . p h p ? o p t i o n=c o m ~ c o n t e n t \& t a s k=v i e w \& i d=776 \& l t e m i d=9 \& n$ w=1\&lang=es\&section-=19 Consultado: 29/3/2008.

\section{Referencias bibliográficas}

BELUCHE, O. (2006) "La separación de Panamá de Colombia. Mitos y falsedades. Reflexiones sobre la patria”. En: Tareas. No. 122 (ene.-ab.). Ciudad de Panamá: CELA, Centro de Estudios Latinoamericanos "Justo Arosemena". Disponible en: http://bibliotecavirtual. clacso.org.ar/ar/libros/panama/cela/tareas/tar122/06beluche.pdf Consultado: 3/12/2007.

CORDERO, A. (2002) Una nación dividida por algo más que un Canal: los dos Panamá. Ciudad de Panamá: UNICEF. Disponible en: www.unicef.org/panama/spanish/LOS_DOS_PANAMA. pdf Consultado: 7/9/2007.

FIGUEROA NAVARRO, A. (1978) Dominio y Sociedad en el Panamá Colombiano: 1821-1903. Ciudad de Panamá: Impresora Panamá, S.A. Disponible en: www.binal.ac.pa/buscar/cldetalle. php?id=138\&from =l Consultado: 3/12/2007.

FUNDACIÓN PARA EL DEBIDO PROCESO - DPLF (2007) Controles y descontroles de la corrupción judicial: Evaluación de la corrupción judicial y de los mecanismos para combatirla en Centroamérica y Panamá. Washington:DPLF. Disponible en: www.dplf.org/uploads/1196091551.pdf Consultado: 11/12/2007.

FUNPADEM (2004) Dimensión y problemática que afecta a los desplazados hacia Ecuador, Venezuela y Panamá, por el conflicto bélico en Colombia. San José: FUNDAPEM.

HUGHES, W. \& QUINTERO, I. (2000) ¿Quiénes son los dueños de Panamá? Versión corregida y ampliada, Panamá: Taller Senda.

PNUD (2003) La igualdad como estrategia de combate a la pobreza en Panamá. Panamá: Programa de las Naciones Unidas para el Desarrollo. Disponible en: www.undp.org.pa/_pnud/ Documents/libro.pdf Consultado: 04/06/2008. 